\title{
Calculations of Oxygen Stability in Lithium-Rich Layered Cathodes
}

\author{
Penghao Xiao, ${ }^{\dagger}$ Z. Q. Deng, ${ }^{\ddagger}$ A. Manthiram, ${ }^{\ddagger}$ and Graeme Henkelman* ${ }^{\dagger}$ \\ ${ }^{\dagger}$ Department of Chemistry and ${ }^{\ddagger}$ Electrochemical Energy Laboratory, Materials Science and Engineering Program, University of Texas \\ at Austin, Austin, Texas 78712, United States
}

ABSTRACT: Oxygen loss can lead to high-capacity $\mathrm{Li}_{2} \mathrm{MnO}_{3}$-based lithiumrich layered cathodes. Substitution of $\mathrm{Mn}$ with other transition metals ( $\mathrm{Ti}$ and Co) significantly affects the amount of oxygen loss and capacity during the first charge/discharge cycle. An explanation of these results is provided with density functional theory $(\mathrm{DFT}+\mathrm{U})$ electronic structure calculations. Oxygen is found to bind more strongly to $\mathrm{Ti}$ and more weakly to Co. The influence of the substitution is attributed to changes of the band gap. Ti lifts the nonbonding band and increases the band gap of the compound, thus raising the energy

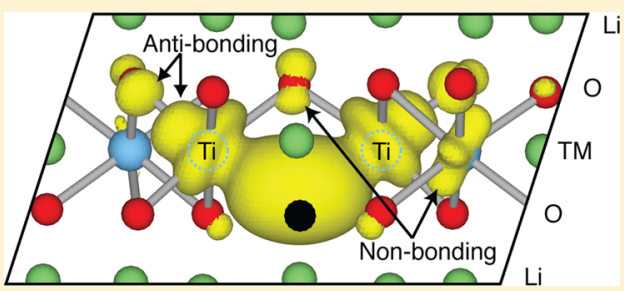
required to redistribute the electrons released upon oxygen loss. Co lowers the nonbonding band and facilitates oxygen loss.

\section{INTRODUCTION}

$\mathrm{Li}_{2} \mathrm{MnO}_{3}$-stabilized $\mathrm{LiMO}_{2}(\mathrm{M}=\mathrm{Mn}, \mathrm{Co}, \mathrm{Ni})$ materials are promising cathodes for $\mathrm{Li}$-ion batteries due to their higher capacity and stability as compared with the parent $\mathrm{LiMO}_{2}$ layered oxides. ${ }^{1}$ The stabilized compounds have a similar structure as $\mathrm{LiMO}_{2}$ except that excess $\mathrm{Li}$ populates the transition-metal layer. This is made clear using the notation, $\mathrm{Li}\left[\mathrm{Li}_{1 / 3} \mathrm{Mn}_{2 / 3}\right] \mathrm{O}_{2}$, where [...] indicates the composition of the transition-metal layer; in this example, $1 / 3$ of the $\mathrm{Mn}$ atoms are replaced with $\mathrm{Li}$. In the end-point material, $\mathrm{Li}_{2} \mathrm{MnO}_{3}, \mathrm{Mn}^{4+}$ cannot be further oxidized. It has been shown that the oxidation of $\mathrm{O}^{2-}$ to $\mathrm{O}_{2}$ compensates for electrons in charging process. ${ }^{2-4}$ For the stabilized materials, it was expected that the compensating electrons would come only from $\mathrm{LiMO}_{2}(\mathrm{M}=$ $\mathrm{Mn}, \mathrm{Co}, \mathrm{Ni}$ ), where $\mathrm{M}$ can be oxidized to a +4 state. Interestingly, oxygen loss is still observed, and it continues after all transition metals are fully oxidized. ${ }^{5,6}$ This oxygen loss results in a higher capacity than either of the end-point materials, so it is important to determine the factors that affect it.

In a previous paper, $\mathrm{Li}\left[\mathrm{Li}_{0.2} \mathrm{Ni}_{0.2} \mathrm{Mn}_{0.6}\right] \mathrm{O}_{2}$ was synthesized, and the influence of different transition-metal ions on oxygen loss during the first cycle was studied. ${ }^{6}$ The results shows that oxygen loss is sensitive to the substitution: introducing Ti into the transition-metal layers reduces oxygen loss, whereas Co promotes it. The explanation provided is that a greater overlap between the $\mathrm{Co}^{3+/ 4+} t_{2 g}$ and $\mathrm{O}^{2-} 2 \mathrm{p}$ bands leads to more metal$\mathrm{O}$ covalency, more delocalized electrons, and a reduced stability of $\mathrm{O}^{2-}$ ions; whereas less overlap between the $\mathrm{Ti}^{3+/ 4+} \mathrm{t}_{2 g}$ and the $\mathrm{O}^{2-} 2 \mathrm{p}$ bands leads to less metal-O covalency, more localized electrons, and an increased stability of $\mathrm{O}^{2-}$ ions. Here we employ density function theory (DFT) calculations to explain the experimental results and test this model from the perspective of oxygen binding and electronic structure calculations.

\section{COMPUTATIONAL METHOD}

To simplify the calculations, we modeled the end-point material $\mathrm{Li}\left[\mathrm{Li}_{1 / 3} \mathrm{Mn}_{2 / 3}\right] \mathrm{O}_{2}$ and then substituted $\mathrm{Mn}$ with other transition metals. All transition metals modeled are in their +4 state, which represents the chemical environment where oxygen loss occurs. DFT $+\mathrm{U}$ calculations were conducted using Vienna $a b$ initio simulation package. ${ }^{12}$ The generalized gradient approximation with PW91 functional was chosen to describe electron exchange and correlation. ${ }^{13}$ All calculations included spin-polarization. Core electrons were incorporated into pseudopotentials with the projector augmented wave method. ${ }^{14,15}$ Valence electrons were described with a plane-wave basis set with an energy cutoff of $400 \mathrm{eV}$. A $2 \times 1 \times 1$ supercell was chosen to represent the periodic crystal. All atoms were allowed to relax, but no gross structural rearrangements were considered. A Monkhorst-Pack $k$-point mesh of $2 \times 4 \times 4$ was set for oxygen binding energy calculations, and a $6 \times 12 \times 12$ mesh was set for density of states (DOS) calculations. An onsite Hubbard term $(U)$ was used for the transition metals to avoid the delocalization of $3 \mathrm{~d}$ electrons as a result of selfinteraction. The effective $U$ values $\left(U_{\text {eff }}=U-J\right)$ were taken from the literature; they are summarized in Table 1. A comparison of band gaps calculated with $\mathrm{DFT}+\mathrm{U}$ and the Heyd-Scuseria-Ernzerhof (HSE06) hybrid functional ${ }^{16}$ is plotted in Figure 1. The HSE band gaps are expected to be in fairly good agreement with experiment, without relying on

Table 1. U Value for Different Transition Metals (TMs)

$\begin{array}{llllll}\mathrm{TM} & U_{\text {eff }} & \text { ref } & \mathrm{TM} & U_{\text {eff }} & \text { ref } \\ \mathrm{Co} & 5.1 & 7 & \mathrm{~V} & 4.0 & 8 \\ \mathrm{Mn} & 5.0 & 9 & \mathrm{Cr} & 3.5 & 10 \\ \mathrm{Ti} & 4.2 & 11 & \mathrm{Fe} & 4.3 & 7 \\ \mathrm{Ni} & 5.96 & 9 & & & \end{array}$

Received: June 15, 2012

Revised: October 1, 2012

Published: October 31, 2012 


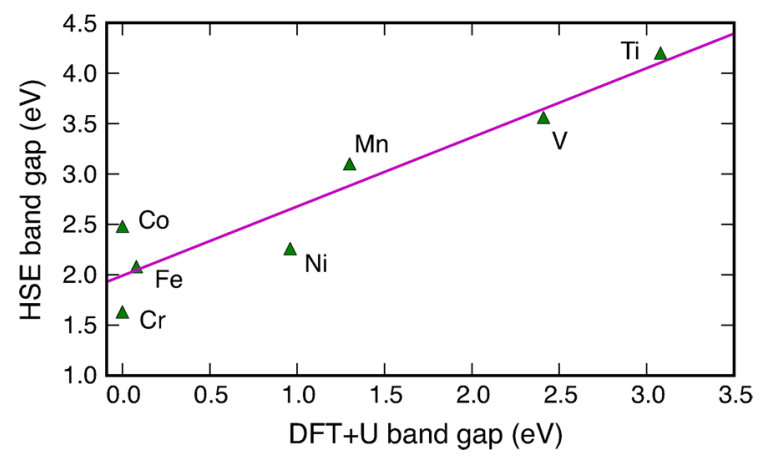

Figure 1. Correlation between band gaps of $\mathrm{Li}_{2} \mathrm{MO}_{3}$ calculated with $\mathrm{DFT}+\mathrm{U}$ and the HSE functional.

any material specific fitting parameters. ${ }^{17}$ Although $\mathrm{DFT}+\mathrm{U}$ underestimates the band gap as compared with HSE, the correlation between the two methods validates the use of DFT $+\mathrm{U}$ and the literature values of $\mathrm{U}_{\text {eff }}$ used for our analysis. Local charges were calculated using a grid-based Bader analysis method. $^{18,19}$

\section{RESULTS}

The effects of $\mathrm{Ti}$ and Co doping on oxygen loss in the first cycle are summarized in Figure $2 .^{6}$ The width of the plateau at

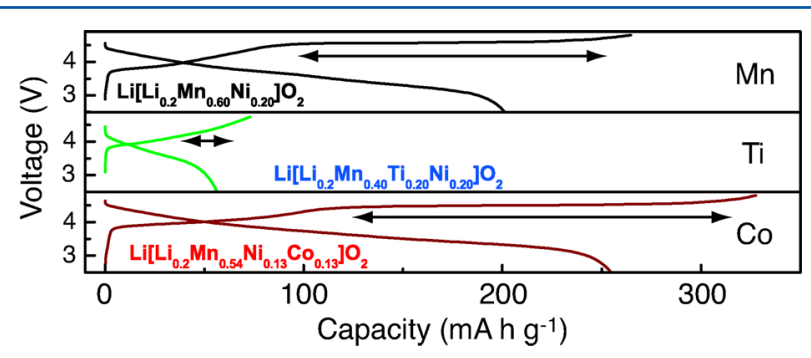

Figure 2. Charge/discharge curves of different compounds from ref 6 showing how $\mathrm{Ti}$ suppresses oxygen loss and Co increases it.

$4.5 \mathrm{~V}$ corresponds to the amount of oxygen loss. When $\mathrm{Mn}$ is substituted for $\mathrm{Ti}$, the plateau increases (indicated by the arrows in the Figure); the opposite is observed for Co substitution.

The oxygen binding energy is a direct indicator of oxygen stability in different compounds. A stronger oxygen-metal bond makes it harder for oxygen to leave the material. Using DFT, the oxygen binding energy is calculated as the energy of the following reaction

$$
\mathrm{Li}_{2 x} \mathrm{M}_{x} \mathrm{O}_{3 x} \rightarrow \mathrm{Li}_{2 x} \mathrm{M}_{x} \mathrm{O}_{3 x-1}+\frac{1}{2} \mathrm{O}_{2}
$$

Our model uses a value of $x=4$ and an oxygen vacancy concentration of $8.3 \%$. The low concentration reduces the interaction of vacancies between periodic images so that the calculations correspond to the onset of oxygen loss, thus simplifying the analysis. The oxygen binding energy of $\mathrm{Li}\left[\mathrm{Li}_{1 / 3} \mathrm{M}_{2 / 3}\right] \mathrm{O}_{2}(\mathrm{M}=\mathrm{Co}, \mathrm{Mn}$, and $\mathrm{Ti})$ is shown in Figure 3. Ti substitution is found to increase the oxygen binding energy, whereas Co decreases it, which agrees with the experimental trends. The average net charge on the oxygen atoms, which is the total electric charge calculated with a Bader analysis minus the number of protons in the nucleus, is also shown to correlate with the oxygen binding energy. This trend has been understood in terms of the degree of covalency between the

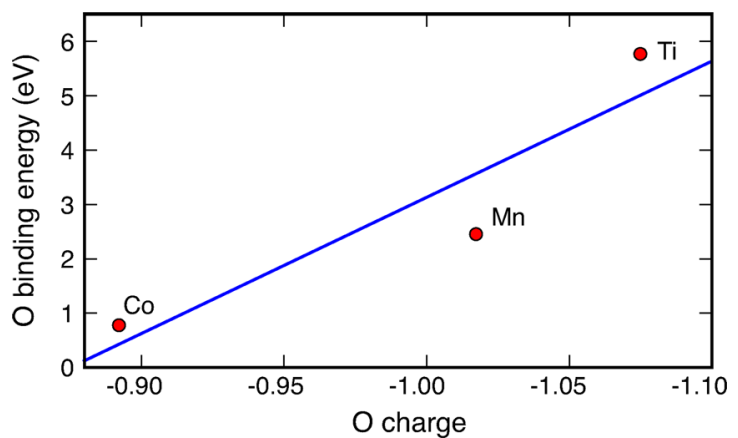

Figure 3. Correlation between oxygen binding energy and the oxygen net charge.

$\mathrm{O}$ and transition metal. ${ }^{6}$ Transition-metal ions that are more electropositive result in more ionic oxygen-metal bonds. When the bonding electrons are relatively more localized on oxygen atoms, these oxygen atoms are difficult to remove, and the material is harder to oxidize. Transition-metal ions that are less electropositive lead to a greater degree of covalency with oxygen and a greater tendency for oxygen loss.

Trends in oxygen stability can be directly understood from the electronic structure of the oxide materials and specifically how the DOS changes with the transition metal, as shown in Figure 4a. One thing worth noting is that the valence bands consist of both oxygen and metal character, with oxygen dominating. There is more metallic contribution in the valence
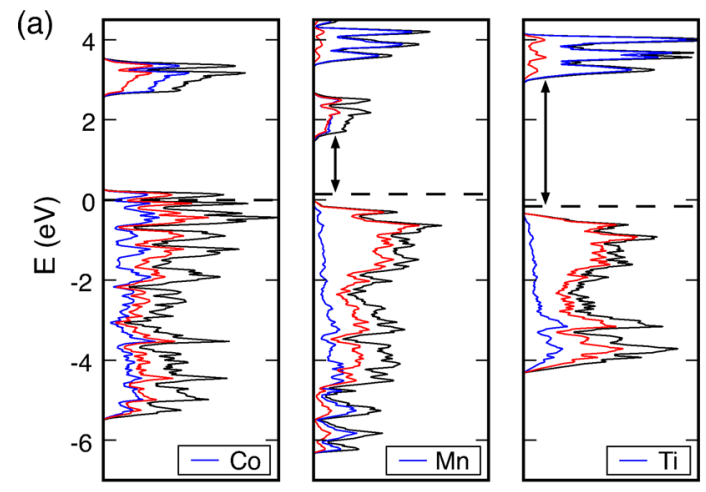

Density of states $N(E)$

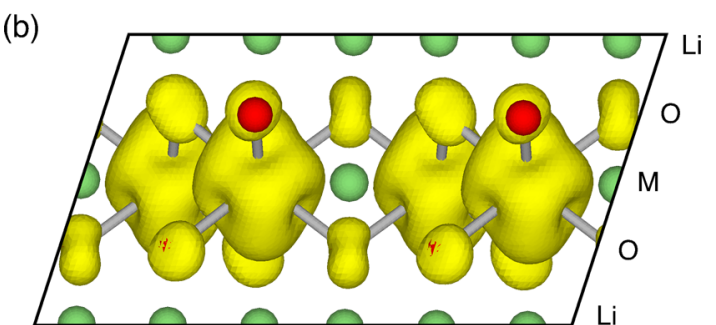

Figure 4. (a) Density of states of $\mathrm{Li}\left[\mathrm{Li}_{1 / 3} \mathrm{M}_{2 / 3}\right] \mathrm{O}_{2}$, where $\mathrm{M}$ is $\mathrm{Co}, \mathrm{Mn}$, or Ti. In each plot, the black curve is the total density; red and blue curves are the partial density on oxygen and the transition metal; and the dashed black line indicates the Fermi level. The band gap increases from left to right, which follows the oxygen binding energy trend (note that $\mathrm{Li}\left[\mathrm{Li}_{1 / 3} \mathrm{Co}_{2 / 3}\right] \mathrm{O}_{2}$ is calculated to be a metal). The energy levels are aligned by the low-lying oxygen $2 \mathrm{~s}$ states. (b) Electron density isosurface (yellow) of the lowest unoccupied molecular orbital (LUMO) in $\mathrm{Li}\left[\mathrm{Li}_{1 / 3} \mathrm{Ti}_{2 / 3}\right] \mathrm{O}_{2}$. The green spheres are $\mathrm{Li}$ and the red spheres are $\mathrm{O}$. The concavity along the bond directions shows the nonbonding character of the LUMO. 
band for Co, which suggests more overlap between the Co and $\mathrm{O}$ bands and a greater covalency. Both $\mathrm{Mn}$ and $\mathrm{Ti}$ have a greater ionic character. In all three compounds, the conduction band primarily consists of nonbonding or antibonding metal states. The nuclear positive charge increases from $\mathrm{Ti}$ to $\mathrm{Co}$ and the metal states are lower in the DOS due to an increased Coulomb attraction. For Co, the nonbonding states overlap with the valence states, and the band gap vanishes.

The band gaps of the three compounds are also found to correlate positively with their oxygen binding energy. This is not surprising: when an $\mathrm{O}$ atom is removed from the material, the localized electrons on the $\mathrm{O}$, which occupied states below the Fermi level, must be accommodated in unoccupied states. The differences in energy for oxygen removal between the different compounds can then be correlated to the band gap. This model is supported by calculations of the DOS of $\mathrm{Li}\left[\mathrm{Li}_{1 / 3} \mathrm{M}_{2 / 3}\right] \mathrm{O}_{2-x}$ shown in Figure 5a. The removal of an $\mathrm{O}$

\section{(a)}
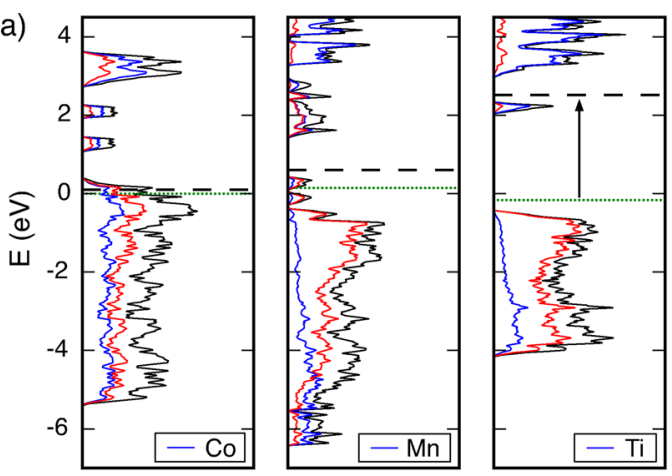

Density of states $\mathrm{N}(\mathrm{E})$

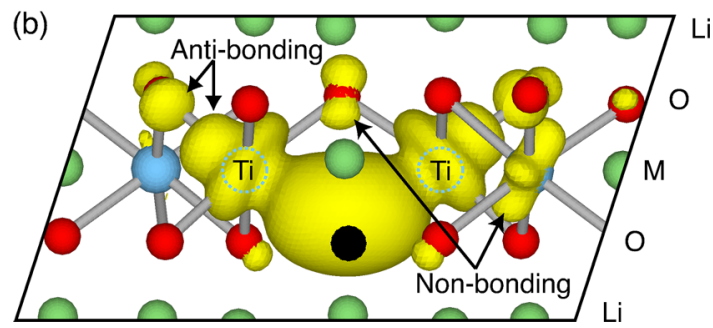

Figure 5. (a) DOS of $\mathrm{Li}\left[\mathrm{Li}_{1 / 3} \mathrm{M}_{2 / 3}\right] \mathrm{O}_{2}$ with an oxygen vacancy in the supercell. The black dashed line shows the increased Fermi level after oxygen removal as compared with before, shown by the green dotted line. (b) Electron distribution of the highest occupied orbital created by oxygen removal in $\mathrm{Li}\left[\mathrm{Li}_{1 / 3} \mathrm{Ti}_{2 / 3}\right] \mathrm{O}_{2}$. Green spheres are $\mathrm{Li}$, red are $\mathrm{O}$; blue $\mathrm{Ti}$; and black is the $\mathrm{O}$ vacancy.

atom releases two electrons to the crystal, which occupy a new state above the Fermi level. In $\mathrm{Li}\left[\mathrm{Li}_{1 / 3} \mathrm{Ti}_{2 / 3}\right] \mathrm{O}_{2-x}$, this new state is localized in an orbital with $\mathrm{d}_{z}{ }^{2}$ character on the two Ti centers neighboring the $\mathrm{O}$ vacancy. The $\mathrm{d}_{z}{ }^{2}$ states on Ti mix with the $\mathrm{p}_{z}$ states of the missing $\mathrm{O}$ in the metal-oxide valence band. The other end of the $\mathrm{Ti}-\mathrm{d}_{z}{ }^{2}$ orbitals point to the neighboring occupied $\mathrm{O}-\mathrm{p}_{z}$ orbitals. The node between the Ti- $\mathrm{d}_{z}{ }^{2}$ and $\mathrm{O}-\mathrm{p}_{z}$ orbitals indicates the antibonding character of the state. A small portion of the state is locating on the second nearest neighbor $\mathrm{Ti}$ atom, with $t_{2 \mathrm{~g}}$ symmetry, which indicates nonbonding character. To summarize, the electrons left behind upon $\mathrm{O}$ loss in $\mathrm{Li}\left[\mathrm{Li}_{1 / 3} \mathrm{Ti}_{2 / 3}\right] \mathrm{O}_{2-x}$ are in a nonbonding/antibonding mixed state.

The DOS also shows why oxygen loss is enhanced upon delithiation. In Figure 6, the removal of a $\mathrm{Li}$ atom in
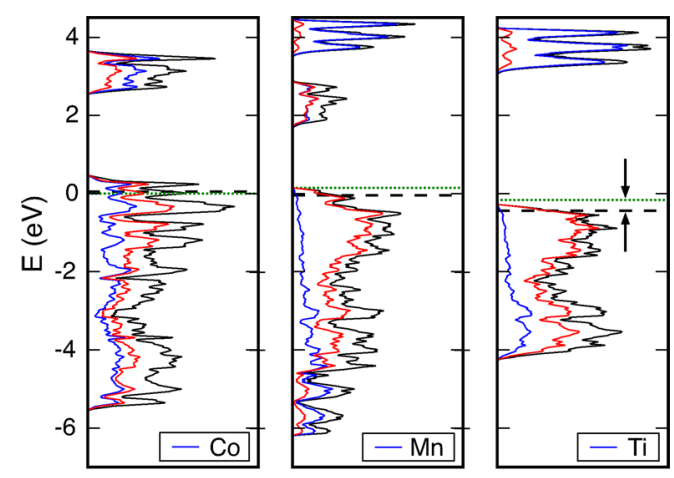

Density of states $\mathrm{N}(\mathrm{E})$

Figure 6. DOS of $\mathrm{Li}\left[\mathrm{Li}_{1 / 3} \mathrm{M}_{2 / 3}\right] \mathrm{O}_{2}$ with a lithium vacancy in the supercell. Fermi levels drop from the green dotted lines to the black dashed lines. All three materials are conductors now.

$\mathrm{Li}_{1-x}\left[\mathrm{Li}_{1 / 3} \mathrm{M}_{2 / 3}\right] \mathrm{O}_{2}$ creates an electronic hole and lowers the Fermi level so that the material becomes a conductor. Because the band gap is zero, oxygen loss is facilitated by the low-lying unoccupied states. This explains why $\mathrm{Li}\left[\mathrm{Li}_{1 / 3} \mathrm{M}_{2 / 3}\right] \mathrm{O}_{2}$ is inactive as a cathode material.

The correlation between the band gap of $\mathrm{Li}\left[\mathrm{Li}_{1 / 3} \mathrm{M}_{2 / 3}\right] \mathrm{O}_{2}$ and the binding of oxygen extends over the first-row transition metals, as shown in Figure 7. These calculations indicate that $\mathrm{Fe}$ and $\mathrm{Cr}$ could be potential doping elements for $\mathrm{Li}$ $\left[\mathrm{Li}_{1 / 3} \mathrm{Mn}_{2 / 3}\right] \mathrm{O}_{2}$, besides $\mathrm{Ni}$ and $\mathrm{Co}$. V should stabilize oxygen as compared with $\mathrm{Mn}$.

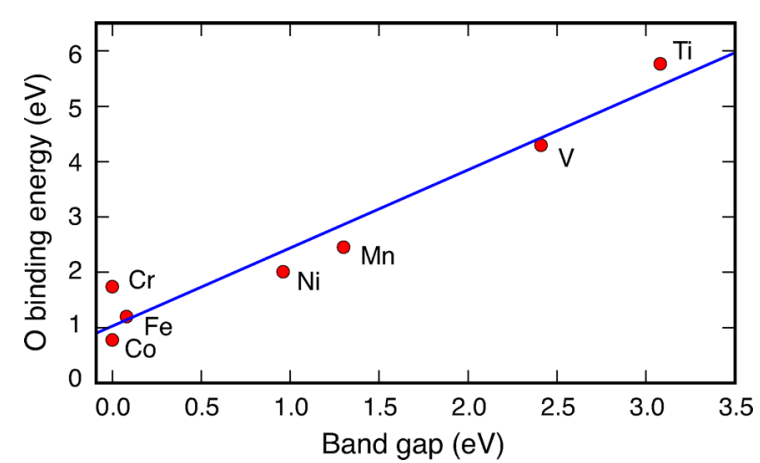

Figure 7. Correlation of oxygen binding in $\mathrm{Li}\left[\mathrm{Li}_{1 / 3} \mathrm{M}_{2 / 3}\right] \mathrm{O}_{2}$ with the band gap. Each band gap is calculated as the energy difference between the bottom of the conduction band and the top of the highest occupied band of oxygen character. This is the conventional band gap for all except the vanadium compound, whose highest occupied states are nonbonding metallic states.

\section{CONCLUSIONS}

We have explained the effect of transition-metal substitutions on oxygen loss in lithium-rich layered oxides from the electronic structure calculated with $\mathrm{DFT}+\mathrm{U}$. The conclusion is that the band gap of the cathode determines the oxygen binding energy because the unoccupied metal bands provide the empty energy levels for electrons from the removed $\mathrm{O}$. Ti substitution increases the band gap because the nonbonding metal band has higher energy due to the weaker nuclear attraction as compared with $\mathrm{Mn}$. Therefore, Ti suppresses oxygen loss. Co substitution decreases the band gap because the low-lying nonbonding metallic band overlaps with the valence band and makes the material a conductor. Therefore, 
Co facilitates oxygen loss. The correlation between band gap and oxygen binding energy is further confirmed by examining other third-period transition metals between $\mathrm{Ti}$ and $\mathrm{Ni}$.

\section{AUTHOR INFORMATION}

\section{Corresponding Author}

*E-mail: henkelman@mail.utexas.edu.

\section{Notes}

The authors declare no competing financial interest.

\section{ACKNOWLEDGMENTS}

This work is supported as part of the program "Understanding Charge Separation and Transfer at Interfaces in Energy Materials (EFRC:CST)" an Energy Frontier Research Center funded by the US Department of Energy, Office of Science, Office of Basic Energy Sciences under award number DESC0001091. Calculations were done with resources from the National Energy Research Scientific Computing Center and the Texas Advanced Computing Center.

\section{REFERENCES}

(1) Thackeray, M. M.; Kang, S.-H.; Johnson, C. S.; Vaughey, J. T.; Benedek, R; Hackney, S. A. J. Mater. Chem. 2007, 17, 3112.

(2) Robertson, A. D.; Bruce, P. G. Chem. Mater. 2003, 15, 19841992.

(3) Yu, D. Y. W.; Yanagida, K.; Kato, Y.; Nakamura, H. J. Electrochem. Soc. 2009, 156, A417.

(4) Pasero, D.; McLaren, V.; Souza, S. D.; West, A. Chem. Mater. 2005, 17, 345-348.

(5) Armstrong, A. R.; Holzapfel, M.; Novák, P.; Christopher; Johnson, S.; Kang, S.-H.; Thackeray, M. M.; Bruce, P. G. J. Am. Chem. Soc. 2006, 128, 8694-8698.

(6) Deng, Z. Q.; Manthiram, A. J. Phys. Chem. C 2011, 115, 70977103.

(7) Chevrier, V. L.; Ong, S. P.; Armiento, R.; Chan, M. K. Y.; Ceder, G. Phys. Rev. B 2010, 82, 075122 .

(8) Scanlon, D. O.; Walsh, A.; Morgan, B. J.; Watson, G. W. J. Phys. Chem. C 2008, 112, 9903-9911.

(9) Hinuma, Y.; Meng, Y. S.; Kang, K.; Ceder, G. Chem. Mater. 2007, 19, 1790-1800.

(10) Wang, L.; Maxisch, T.; Ceder, G. Phys. Rev. B 2006, 73, 195107.

(11) Morgan, B. J.; Watson, G. W. Surf. Sci. 2007, 601, 5034-5041.

(12) Kresse, G.; Hafner, J. Phys. Rev. B 1993, 47, R558-R561.

(13) Perdew, J. P.; Wang, Y. Phys. Rev. B 1992, 45, 13244-13249.

(14) Blöchl, P. E. Phys. Rev. B 1994, 50, 17953.

(15) Kresse, G.; Joubert, D. Phys. Rev. B 1999, 59, 1758.

(16) Heyd, J.; Scuseria, G. E.; Ernzerhov, M. J. Chem. Phys. 2006, 124, 219906.

(17) Marsman, M.; Paier, J.; Stroppa, A.; Kresse, G. J. Phys.: Condens. Matter 2008, 2222, 064201.

(18) Bader, R. F. W. Atoms in Molecules: A Quantum Theory; Oxford University Press: New York, 1990.

(19) Tang, W.; Sanville, E.; Henkelman, G. J. Phys.: Condens. Matter 2009, 21, 084204 . 